\title{
Allogeneic Peripheral Blood Stem Cell Transplantation as an Alternative to Allogeneic Bone Marrow Transplantation
}

Since the early 1990's, the use of peripheral blood stem cells (PBSC) for autologous transplantation following marrow-ablative chemotherapy has been replacing autologous bone marrow transplantation (auto-BMT) for the treatment of hematologic malignancy and solid tumors (1). Autologous PBSC transplantation (auto-PBSCT) provides several advantages over autoBMT, including 1) rapid hematopoietic engraftment, 2) less requirement for blood transfusion, antibiotic therapy and hospitalization days, and 3) avoidance of .risks and discomfort associated with general anesthesia for collecting hematopoietic stem cells (2). Taking these advantages into consideration, the use of PBSC was applied for syngeneic and allogeneic transplantation in the early 1990's. Subsequently, allogeneic PBSC transplantation (allo-PBSCT) has been increasingly used for the treatment of hematologic malignancy (3). Recent clinical evidences clearly indicate that allo-PBSCT can be used as an alternative to allogeneic BMT (allo-BMT) $(4,5)$. Similarly to auto-PBSCT, rapid engraftment of neutrophils and/or platelets has been assessed after allo-PBSCT in many clinical trials. Since PBSC allografts contain 10-fold more T lymphocytes than BM allografts, there is a major concern about the increased risk of graft-vs-host disease (GVHD). However, a significant increase in the incidence or severity of acute GVHD has not been demonstrated. In contrast, there are some reports which claim a relatively high incidence of chronic GVHD (6).

Recently we reported a Japanese multicenter pilot study of allo-PBSCT for the treatment of standard-risk leukemia (7). Twenty-two patients with standard-risk leukemia were transplanted with granulocyte-colony stimulating factor (G-CSF)mobilized PBSC from an HLA-identical sibling donor, and received cyclosporine and methotrexate for GVHD prevention. Median days to reach an absolute neutrophil count of $>500 / \mu \mathrm{l}$ and platelets $>50,000 / \mu \mathrm{l}$ were $12(9-20)$ and $16(11-$ 32 ), respectively. Grade II-IV acute GVHD developed in 6/21 (29\%) and extensive chronic GVHD in 12/20 (60\%). Thus, our observations also indicate that allo-PBSCT is characterized by rapid hematologic engraftment, no increase of acute GVHD and an increased risk of chronic GVHD, and can be used as an alternative to allo-BMT.

At least more than $50 \%$ of patients with acute myelogenous leukemia, acute lymphoblastic leukemia and chronic myelogenous leukemia can be cured by treatment with allo-BMT when they are transplanted during the first remission or chronic phase. In the setting of allo-BMT, the cure of leukemia is due to the eradication of residual leukemic cells provided by the pretransplant marrow-lethal chemoradiotherapy or chemo- therapy and the antileukemic effects associated with acute and/ or chronic GVHD. These antileukemic effects of allo-BMT are referred to as graft-versus-leukemia (GVL) effects and are mediated predominantly by donor-derived T cells. Similar to the antileukemic effects, graft-versus-lymphoma (GVLy) effects are also elucidated by clinical observations; a prospective randomized trial of auto-BMT versus allo-BMT demonstrated that both the relapse-free and progression-free survivals are significantly higher in allo-BMT than in auto-BMT (8). Since PBSC allografts are enriched with T cells and NK cells, and allo-PBSCT produces acute and chronic GVHD as frequently as or more frequently than allo-BMT, GVLy effects can be highly expected after allo-PBSCT. Accordingly, we used alloPBSCT from an HLA-identical sibling donor in the treatment of a patient with advanced follicular lymphoma, who developed leukemic change and refractoriness to the intensive chemotherapy for acute lymphoblastic leukemia (9).

See also p 1050.

The patient achieved complete remission (CR) and is now maintaining $\mathrm{CR}$ without any posttransplant treatment for more than 2 years after allo-PBSCT. Thus, our clinical experience suggests that allo-PBSCT may be a choice for treatment for refractory or bad-prognosis lymphoma when an HLA-identical donor is obtained. Further studies with more patients and a longer follow-up time will be necessary to assess the efficacy of this new treatment modality.

Mine Harada, MD The Department of Internal Medicine II, Okayama University Medical School, 2-5-1 Shikata-cho, Okayama 700-8558

\section{References}

1) Gratwohl A, Passweg J, Baldomero H, Herman J, and European Group for Blood and Marrow Transplantation (EBMT). Blood and marrow transplantation activity in Europe 1996. Bone Marrow Transplant 22: 227240, 1998.

2) Schmitz N, Linch DC, Dreger P, et al. Randomized trial of filgrastimmobilised peripheral blood progenitor cell transplantation versus autologous bone marrow transplantation in lymphoma patients. Lancet 347: $353-357,1996$.

3) Harada M. Allogeneic peripheral blood stem cell transplantation is coming of age. Int J Hematol 62: 1-5, 1995 (Editorial).

4) Bensinger WI, Clift R, Martin P, et al. Allogeneic peripheral blood stem cell transplantation in patients with advanced hematologic malignancies: A retrospective comparison with marrow transplantation. Blood 88: 
2794-2800, 1996

5) Schmitz N, Bacigalupo A, Hasenclever D, et al. Allogeneic bone marrow transplantation vs filgrastim-mobilised peripheral blood progenitor cell transplantation in patients with early leukaemia: first results of a randomised multicentre trial of the European Group for Blood and Marrow Transplantation. Bone Marrow Transplant 21: 995-1003, 1998.

6) Storek J, Gooley T, Siadak M, et al. Allogeneic peripheral blood stem cell transplantation may be associated with a high risk of chronic graft-versushost disease. Blood 90: 4705-4709, 1997.

7) Harada M, Shinagawa K, Kawano F, et al. Allogeneic peripheral blood stem cell transplantation for standard-risk leukemia. A multicenter pilot study: Japanese experience. Bone Marrow Transplant 21 Suppl 3: S54S56, 1998.

8) Jones RJ, Ambinder RF, Piantadose S, Piantadosis S, Santos GW. Evidence of a graft-versus-lymphoma effect associated with allogeneic bone marrow transplantation. Blood 77: 649-653, 1991.

9) Masuda R, Teshima $T$, Ishimaru F, et al. Allogeneic peripheral blood stem cell transplantation for the treatment of refractory follicular lymphoma. Intern Med 37: 1050-1054, 1998. 